\title{
Cucurbitacin B Diminishes Metastatic Behavior of Cholangiocarcinoma Cells by Suppressing Focal Adhesion Kinase
}

\author{
Putthaporn Kaewmeesri, Veerapol Kukongviriyapan, Auemduan Prawan, \\ Sarinya Kongpetch, Laddawan Senggunprai*
}

\begin{abstract}
Objective: Cholangiocarcinoma (CCA) is a malignant tumor with aggressive metastatic property resulted from dysregulation of metastasis-regulated signaling pathways. The aim of this study was to investigate the effect of cucurbitacin B on metastatic behavior of CCA cells through modulation of focal adhesion kinase (FAK) protein. Methods: KKU-452 cells were treated with a specific FAK inhibitor, FAK inhibitor-14, or cucurbitacin B at various concentrations for $24 \mathrm{~h}$. Cell viability was assessed by sulforhodamine B assay. The migratory and invasive abilities of the cells were investigated using wound healing and transwell invasion assays, respectively. The fibronectin-coated plate was used for adhesion assay. The effects of the test compounds on FAK activation and the expression of metastasis-associated proteins were determined by Western blot analysis. The amount of MMP-9 was evaluated using a commercial ELISA Kit. Results: FAK inhibitor-14 and cucurbitacin B at concentrations which minimally affected KKU-452 cell viability could suppress FAK activation, evidently by decreased level of phospho-FAK protein after exposure to the compound. At these conditions, cucurbitacin B suppressed metastatic behavior including migration, invasion and adhesion abilities of CCA cells similar to FAK inhibitor-14. Further molecular studies demonstrated that FAK inhibitor-14 and cucurbitacin B downregulated the expression of metastasis-associated proteins including MMP-9, ICAM-1 and VEGF. Consequently, exposure to cucurbitacin B inhibited the production of MMP-9 enzyme in CCA cells similar to FAK inhibitor-14 treatment. Conclusion: FAK participated in regulation of metastatic behavior of KKU-452 CCA cells. Cucurbitacin B suppressed FAK activation in the cells which was associated with inhibition of metastasis essential steps and their related metastatic proteins. The compound may be developed as a novel therapeutic agent for CCA metastasis therapy.
\end{abstract}

Keywords: FAK- cholangiocarcinoma- metastasis- cucurbitacin B- FAK inhibitor- MMP-9- ICAM-1- VEGF

Asian Pac J Cancer Prev, 22 (1), 219-225

\section{Introduction}

Cholangiocarcinoma (CCA) or bile duct cancer is a malignant tumor with extremely aggressive metastatic behavior resulting in a very poor prognosis (Doherty et al., 2017). Chronic inflammation by liver fluke infection is one of the established risk factors associated with cholangiocarcinogenesis particularly for people residing in Southeast Asia (Zheng et al., 2017). According to changes in intracellular signaling networks contributing in CCA development, targeted therapies to these pathways have been intensively studied for eventually gaining promising treatment of CCA (Fouassier et al., 2019).

Metastasis of cancer is an important clinical problem for cancer treatment. In CCA, most patients are diagnosed at an advanced or metastatic stage, where curative surgical treatment is not appropriate and chemotherapy is a remaining option. Prognosis for patients with metastatic cancer is generally poor. Recently, the association between metastasis and survival rate of CCA patients in Eastern region of Thailand has been analyzed. The results showed that metastasis was a significant clinical predictor of death among these patients (Charonpongsuntorn et al., 2019). It is therefore, effective therapeutic compounds capable of suppressing CCA metastasis are still highly needed. Cancer cell metastasis depends on the ability of cells to migrate and invade in which they are influenced by the surrounding extracellular matrix (ECM) (Welch and Hurst, 2019). Thus, degradation of ECM components considered as an essential step in facilitating the metastasis process. It has been reported that matrix metalloproteinase-9 (MMP-9) is the major enzyme participating in proteolytic degradation of ECM proteins particularly type IV collagen (Curran and Murray, 2000). Besides migratory and invasive properties, 
the adhesion ability of cells to ECM is also required for cancer cells with metastatic behavior to be settled at the target organ site (Paschos et al., 2009). Additionally, angiogenesis, the establishment of new blood vessels, is also a necessary component of the metastatic pathway. The spread of metastatic cells depends on an adequate supply of oxygen and nutrients from the vascular network formed (Bielenberg and Zetter, 2015). Hence, the interventions targeting on cancer cell migration, invasion, adhesion as well as angiogenesis could be the potential strategies for suppression of cancer metastasis. It has been reported that several compounds could inhibit these metastaticassociated events and molecules such as ellagic acid inhibited MMP-9 expression in human prostate cancer cells (Pitchakarn et al., 2013) and trimellitic acid had the capability of angiogenic inhibition by suppressing proangiogenic activity of fibroblast growth factor 2 and inhibiting HUVEC tube formation (Mahdavi et al., 2017).

Metastasis process is regulated by several intracellular signaling cascades (Inan and Hayran, 2019). Cumulative evidence suggested that FAK acts as a prominent regulator molecule for transmitting signals to activate downstream targets associated with metastasis process (Tai et al., 2015). Elevation of FAK expression was strongly associated with tumor metastasis. The activation of FAK increased the expression of metastasis-associated molecules such as MMPs and intercellular adhesion molecule-1 (ICAM-1) in many cancer cells (Hauck et al., 2001; Schlaepfer et al., 2004; Taglia et al., 2007). Additionally, FAK has been implicated in the regulation of tumor angiogenesis through promoting vascular endothelial growth factor (VEGF) expression and activity (Peng et al., 2004). Therefore, suppression of FAK has been one of therapeutic interest strategies for treatment or inhibition of cancer metastasis.

Bioactive phytochemical compounds isolated from natural products have been focused as a potential option for cancer treatment (Pitchakarn et al., 2013; Zakaria et al., 2019). Cucurbitacin B is a tetracyclic triterpenoid derived from edible cucurbitaceous plants (Patlolla and Rao, 2012). Our previous studies demonstrated that this compound exerted antiproliferative and apoptosis-inducing effects on KKU-100 CCA cells via suppression of FAK-mediated signaling pathways (Klungsaeng et al., 2019; Klungsaeng et al., 2020). In this study, we aimed to investigate the effect of cucurbitacin B on metastatic behavior of CCA cells through modulation of FAK protein. KKU-452 CCA cell line, a newly established CCA cells derived from CCA patients with liver fluke infection with remarkably high migration and invasion properties (Saensa-Ard et al., 2017), was employed in the study.

\section{Materials and Methods}

\section{Materials}

Reagents for cell culture were purchased from Gibco-BRL Life Technologies (Grand Island, New York). Selective FAK inhibitor (FAK inhibitor-14), cucurbitacin B and sulforhodamine B were obtained from Sigma Chemical (St. Louis, MO). The primary antibody against phospho-FAK was purchased from Cell Signaling Technology (Dancer, MA, USA). The primary antibodies against FAK, MMP-9, ICAM-1, VEGF and $\beta$-actin and the secondary horseradishperoxidase (HRP)-linked antibodies were obtained from Santa Cruz Biotechnology, Inc. (California, USA).

\section{Cell line and cell culture}

A human CCA cell line, KKU-452, was kindly provided by the Cholangiocarcinoma Research Institute, Khon Kaen University. The cells were grown in Ham's F12 media containing 10 mM HEPES (pH 7.3), 10\% fetal calf serum and antibiotics $(100 \mathrm{U} / \mathrm{mL}$ penicillin and 100 $\mu \mathrm{g} / \mathrm{mL}$ gentamicin). Cells were grown in a humidified atmosphere of $5 \% \mathrm{CO}_{2}$ at $37^{\circ} \mathrm{C}$ and subcultured using $0.25 \%$ trypsin-EDTA every 2 days.

\section{Cytotoxicity assay}

The effects of test compounds on the viability of KKU-452 CCA cells were assessed using sulforhodamine B colorimetric assay. Briefly, the cells were exposed to various concentrations of test compounds for $24 \mathrm{~h}$. Thereafter, the cells were fixed with $10 \%$ trichloroacetic acid for $1 \mathrm{~h}$ at $4^{\circ} \mathrm{C}$ and subsequently stained with $0.4 \%$ sulforhodamine $\mathrm{B}$ in $1 \%$ acetic acid for $30 \mathrm{~min}$. Unbound dye was discarded by rinsing with $1 \%$ acetic acid, and the protein-bound dye was then dissolved in $10 \mathrm{mM}$ Tris solution. The color intensity was quantified at $540 \mathrm{~nm}$ using a microplate spectrophotometer.

\section{Western blot analysis}

KKU-452 cells were treated with different concentrations of test compounds for indicated time points. After treatment, whole cell lysates were prepared using ice-cold RIPA lysis buffer (Thermo Scientific, USA) following the manufacturer's instructions. The $30 \mu \mathrm{g}$ of proteins were then subjected to $10 \%$ SDS-PAGE and electrophoretically transferred onto a PVDF membrane. Non-specific sites on the membranes were blocked with $5 \%$ bovine serum albumin in TBS buffer containing $0.1 \%$ Tween-20 for $1 \mathrm{~h}$. Thereafter, the membranes were incubated with specific primary antibodies at $4^{\circ} \mathrm{C}$ for an overnight. After washing, the membranes were subsequently incubated with HRP-conjugated secondary antibody at room temperature for $2 \mathrm{~h}$. The target proteins were detected using chemiluminescence detection system (LuminataTM Forte Western HRP Substrate, Merck Millipore, Watford, UK) and visualized by ChemiDocTM MP Imaging system.

\section{Wound healing assay}

The migratory ability of KKU-452 cells was evaluated using wound healing assay. Briefly, the cells were cultured in a $24-w e l l$ plate to achieve $90-100 \%$ confluency. A linear wound then was made using a sterile $200 \mu \mathrm{L}$ pipette tip. The unattached cells were removed by washing with PBS. The cells were subsequently treated with various concentrations of test compounds and the wounds were photographed at 0 and $24 \mathrm{~h}$ of treatment using a phase-contrast microscope. The migration ability was assessed from the closing of the scratched wound which was determined using Image-Pro Plus software (Media Cybernetics, LP, USA). 


\section{Invasion assay}

The KKU-452 cells suspended in serum-free medium containing vehicle or various concentrations of test compounds were placed in the upper compartment of Transwell Boyden chamber (Corning, Lowell, MA) pre-coated with $0.3 \mathrm{mg} / \mathrm{mL}$ Matrigel (BD Biosciences, Bedford, MA). The bottom chamber was supplemented with $10 \%$ fetal calf serum-containing media. After incubation for $24 \mathrm{~h}$, the non-invaded cells on the upper surface were scraped using a cotton swab. The invaded cells on the lower side of the membrane were fixed with methanol and stained with crystal violet. The stained cells were then photographed and the number of invaded cells were quantified.

\section{Cell adhesion assay}

The cell adhesion assay was carried out as described previously (Barni et al., 2012) with slightly modification. Briefly, a 96-well plate was coated with $100 \mu \mathrm{L}$ of $0.64 \mu \mathrm{g} /$ $\mathrm{mL}$ fibronectin and incubated for $1 \mathrm{~h}$ at room temperature. The $1 \%$ bovine serum albumin was then added to block the non-specific binding sites. The pre-coated plate was incubated for $2 \mathrm{~h}$ at $37^{\circ} \mathrm{C}$ and then washed twice with PBS. Thereafter, the KKU-452 cells in serum free media containing various concentrations of test compounds were placed on the fibronectin-pre-coated plate and allowed to adhere for $30 \mathrm{~min}$ at $37^{\circ} \mathrm{C}$. After that, unattached cells were removed and adherent cells were fixed with methanol for $20 \mathrm{~min}$ at $4^{\circ} \mathrm{C}$, stained with $0.2 \%$ crystal violet in $2 \%$ ethanol for $10 \mathrm{~min}$ at room temperature, and then photographed. Subsequently, the dye was solubilized
Cucurbitacin B Suppresses Cholangiocarcinoma Cells Metastasis with $5 \%$ acetic acid in $10 \%$ methanol and quantified by measuring the absorbance at $540 \mathrm{~nm}$ using a microplate reader.

\section{Determination of MMP-9 level}

The KKU-452 cells were treated with various concentrations of test compounds for $24 \mathrm{~h}$. Thereafter, the cultured cells were trypsinized, washed with PBS and adjusted to $1 \times 10^{6}$ cells for each reaction. The cells were then completely lysed using an ultrasonic cell disruptor and centrifuged to pellet the cellular debris. The supernatants were collected to quantify the amount of MMP-9 using Human MMP-9 ELISA Kit (Elabscience, Houston, Texas) according to the manufacturer's instructions.

\section{Statistical analysis}

The data were analyzed using One-way analysis of variance with post hoc Student-Newman-Keuls (SNK) test. Results were considered to be statistically significant with a P value less than 0.05 . The statistical analyses were employed using SigmaStat v.3.5 Program (Systat Software Inc., San Jose, California).

\section{Results}

The effects of FAK inhibitor and cucurbitacin B on CCA cell viability

The effects of FAK inhibitor-14 and cucurbitacin B on KKU-452 CCA cell viability were determined by the sulforhodamine B assay. The cells were exposed to various concentrations of the test compounds for $24 \mathrm{~h}$. The results

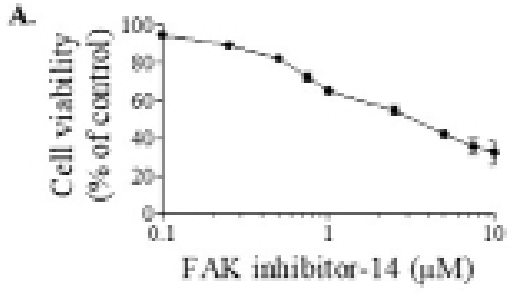

c.
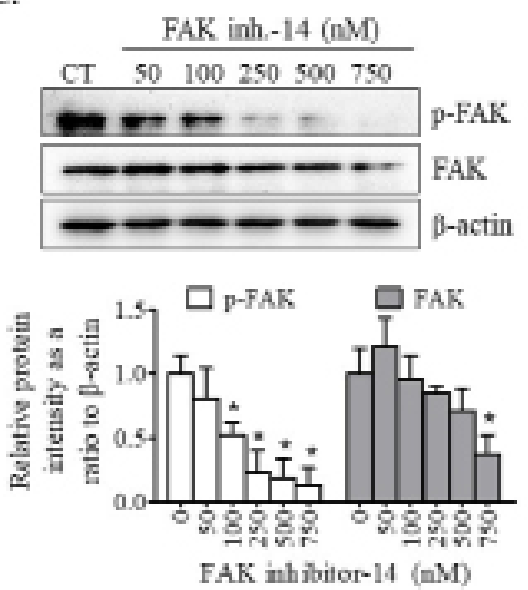

B.

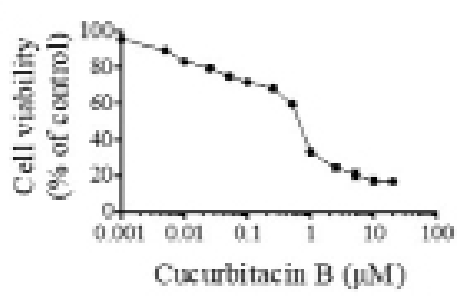

D.

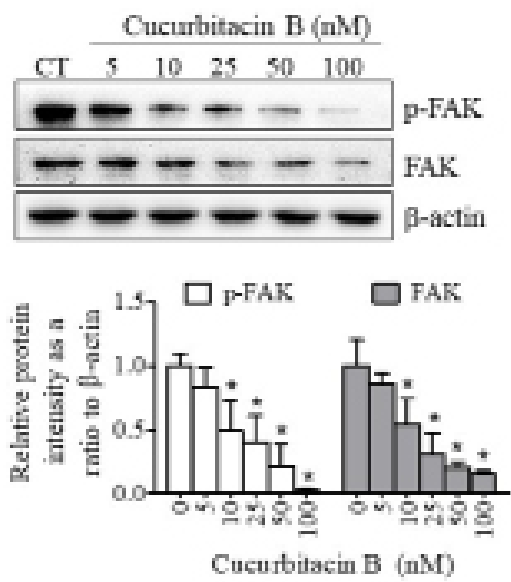

Figure 1. Effects of FAK Inhibitor and Cucurbitacin B on Cell Viability and FAK Activation in CCA Cells. KKU-452 cells were treated with designated compounds for $24 \mathrm{~h}$. Thereafter, the cell viability of (A) FAK inhibitor-14- and (B) cucurbitacin B-treated cells was evaluated using sulforhodamine B assay. Activation of FAK in cells treated with (C) FAK inhibition-14 or (D) cucurbitacin B was determined using Western blot analysis. Representative images and quantification graphs are shown. Data are expressed as mean $\pm \mathrm{SD}$ of three independent experiments. $*$, p $<0.05$ vs respective control. 
A.
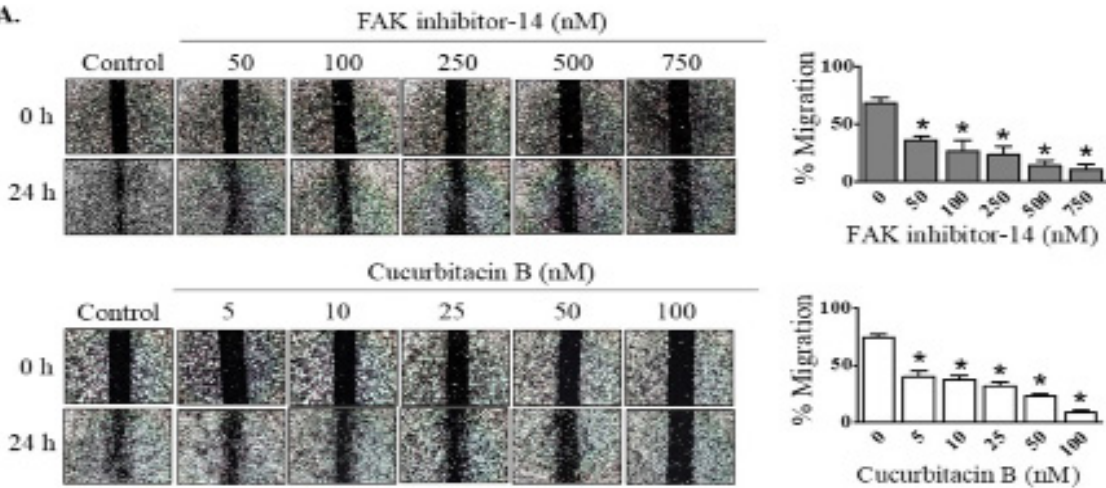

FAK inhibitor-14 (nM)

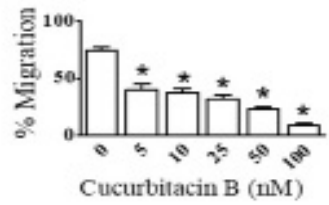

B.
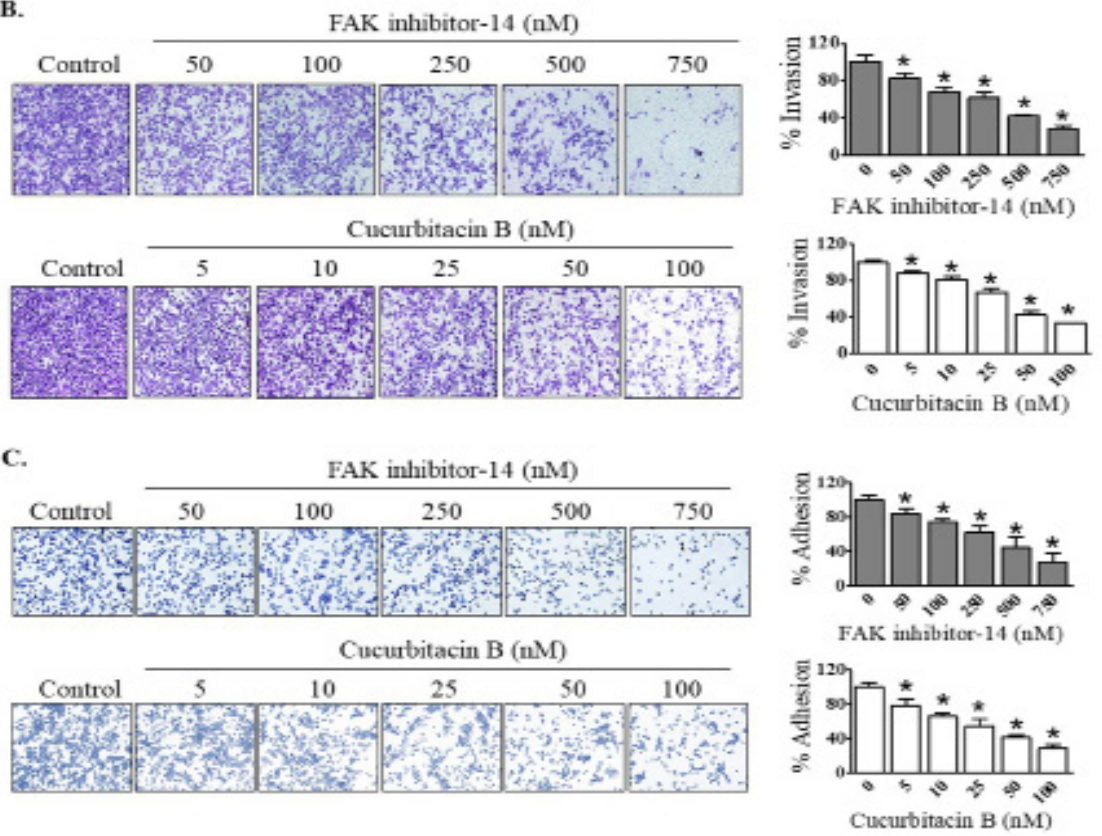

Figure 2. Effects of FAK Inhibitor and Cucurbitacin B on Metastatic Behavior of CCA Cells. KKU-452 cells were exposed to FAK inhibitor-14 or cucurbitacin B at various concentrations. After that, (A) migratory, (B) invasive and (C) adhesion abilities were assessed. Representative figures and quantification graphs are shown. Data represent mean \pm SD of three independent experiments. ${ }^{*}, \mathrm{p}<0.05$ vs respective control.

showed that IC50 values of cytotoxicity effects on KKU452 cells of FAK inhibitor-14 and cucurbitacin B were $3.64 \pm 0.09$ and $0.66 \pm 0.02 \mu \mathrm{M}$, respectively (Figures $1 \mathrm{~A}$ $\& 1 \mathrm{~B})$. Low concentrations of these compounds which negligibility or minimally affected the cell viability were used for further studies.

\section{Cucurbitacin B suppressed FAK activation similar to FAK inhibitor}

Given that FAK acts as a regulator molecule for transmitting signals to activate downstream targets associated with metastasis processes (Tai et al., 2015). We firstly evaluated the effects of FAK inhibitor and cucurbitacin B on FAK activation in KKU-452 cells. The results showed that FAK inhibitor-14 suppressed the phosphorylation of FAK, although FAK protein level was not significantly affected (Figure 1C). Similarly, cucurbitacin B could significantly inhibit FAK activation, evidently by decreased level of phospho-FAK protein after exposure to the compound. Moreover, cucurbitacin B also suppressed the expression of FAK protein (Figure 1D).
Cucurbitacin B diminished metastatic behavior of CCA cells similar to FAK inhibitor

It has been reported that FAK is a key regulator of metastasis processes in several cancer cells. In this study, we investigated whether FAK participates in regulation of metastatic behavior of KKU-452 CCA cells. The effects of FAK inhibitor-14 on migratory, invasive and adhesion abilities of KKU-452 cells were then examined. The results clearly demonstrated that FAK inhibitor-14 significantly suppressed migration, invasion as well as adhesion of CCA cells in a concentration-dependent manner (Figure 2). Similar to FAK inhibitor, cucurbitacin $\mathrm{B}$ could exert anti-migratory, -invasive and -adhesive activities against CCA cells in a dose-dependent manner (Figure 2).

Cucurbitacin B suppressed MMP-9 similar to FAK inhibitor

MMP-9 is a main proteolytic enzymes involving in degradation of basement membranes. We subsequently determined the effect of FAK inhibitor-14 on MMP-9 
A.

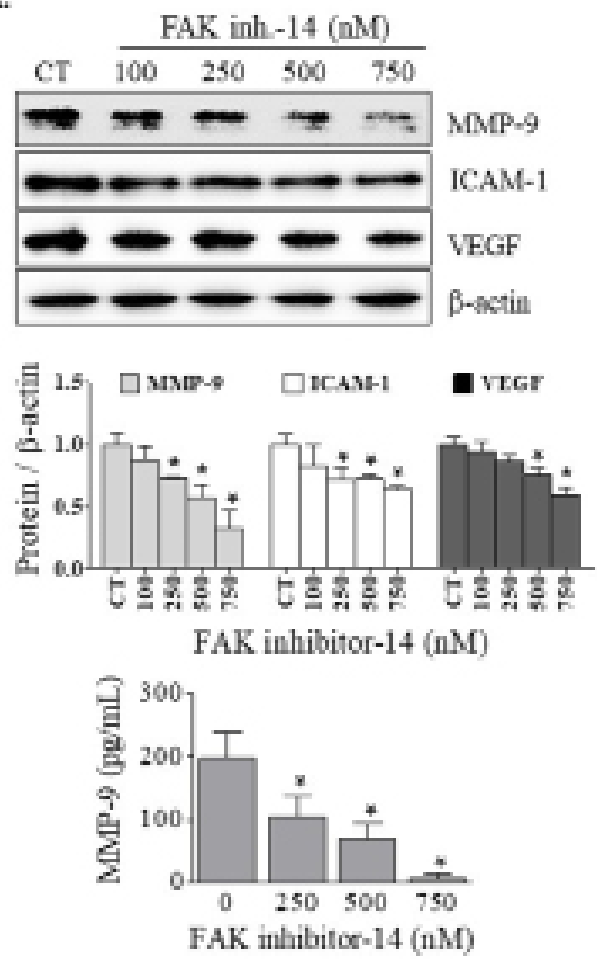

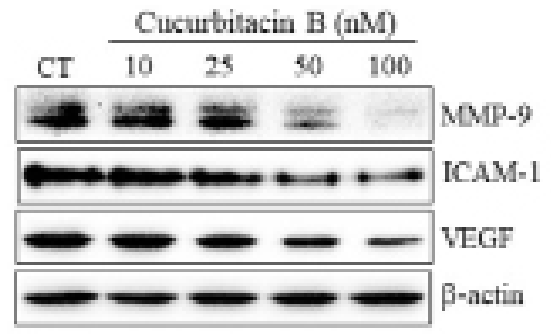

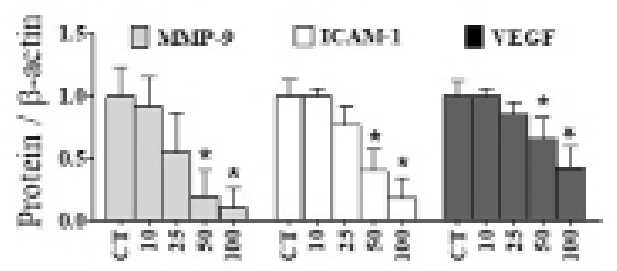

Cucurbitacin $\mathrm{B}(\mathrm{nM})$

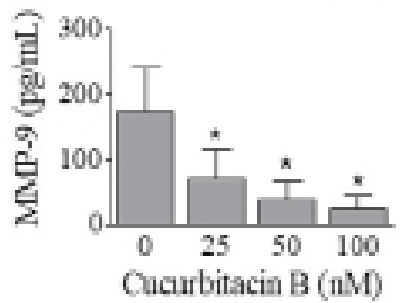

Figure 3. Effects of FAK Inhibitor and Cucurbitacin B on Metastasis-Related Molecules. KKU-452 cells were incubated with (A) FAK inhibitor-14 or (B) cucurbitacin B at various concentrations for $24 \mathrm{~h}$. Afterward, whole cell lysates were prepared for determination of the expression of metastasis-associated proteins using Western blot analysis. Representative figures and quantification graphs are shown. Quantitative determination of MMP-9 concentration in cell lysates was performed using ELISA kit assay. Data are mean \pm SD averaged from three independent experiments. $*, p<0.05$ vs respective control.

expression in KKU-452 cells. Additionally, quantitative determination of MMP-9 concentration was also performed. The results showed that FAK inhibitor-14 significantly suppressed MMP-9 expression in a dose-dependent manner, which led to a decrease of MMP-9 level in CCA cells (Figure 3A). Likewise, exposure to cucurbitacin $\mathrm{B}$ also inhibited the expression of MMP-9 protein. Consequently, the amount of MMP-9 was substantially decreased by cucurbitacin B treatment (Figure 3B).

Cucurbitacin B downregulated metastasis-associated molecules similar to FAK inhibitor

Apart from MMP-9, the effect of FAK inhibitor-14 on the expression of other metastasis-associated proteins, ICAM-1 and VEGF, was also determined. It was found that the expression of these two proteins was downregulated by the inhibitor treatment (Figure 3A). Similar to FAK inhibitor, cucurbitacin B significantly suppressed the expression of ICAM-1 and VEGF in a dose-dependent manner (Figure 3B).

\section{Discussion}

The signaling pathways regulating metastasis process and the metastatic-associated proteins have been targeted for metastasis inhibition. For decades, phytochemical from fruits and vegetables have gained increased attention for development as anti-metastatic agents (Pitchakarn et al., 2013; Tuponchai et al., 2019). In the present study, it was demonstrated that cucurbitacin B suppressed FAK activation in KKU-452 CCA cells which was associated with inhibition of metastasis essential steps and their related metastatic proteins. Thus, this phytochemical compound may be a prospective candidate for the treatment of CCA metastasis.

Given the critical role of FAK in metastatic cascade, suppression of FAK activation could be a potential therapeutic strategy for prevention or inhibition of cancer metastasis (Lee et al., 2015; Tai et al., 2015). In CCA, enhanced FAK expression has been detected which was well correlated with cancer aggressiveness and poor clinical outcome of patients (Khansaard et al., 2014). Herein, the role of FAK contributing in CCA metastasis was confirmed using a specific FAK inhibitor, FAK inhibitor-14. Exposure of the inhibitor to high metastatic potential CCA cells, KKU-452, resulted in suppression of metastatic behavior of the cells. Moreover, FAK inhibitor also diminished the expression of metastasisand angiogenesis-associated proteins including MMP-9, ICAM-1 and VEGF. These data highlighted that FAK protein positively regulates CCA metastasis.

Several natural active ingredients exerted their antimetastasis activities against cancer cells via inactivation of FAK activity (Sun et al., 2015; Masraksa et al., 2020). Previous studies have demonstrated that the anti-metastasis effect of cucurbitacin B against breast cancer cells was mediated through FAK suppression (Luo et al., 2018; Liang et al., 2019). In the present study, the results showed that cucurbitacin B inhibited FAK activation in KKU-452 
cells by alleviating the expression of both phospho-FAK and FAK proteins. The data suggested that cucurbitacin $\mathrm{B}$ might decrease expression or decrease phosphorylation of FAK in KKU-452 cells, which subsequently led to FAK inhibition. It is noted that cucurbitacin $\mathrm{B}$ had a more potent inhibitory effect on FAK protein than that of the specific FAK inhibitor-14. FAK activation has an impact on the metastatic behavior of cancer cells (Schlaepfer et al., 2004). As expected, after suppression of FAK in CCA cells by cucurbitacin B, the essential events of cancer metastasis, including migration, invasion and adhesion, were consequently inhibited. Furthermore, activation of FAK is an important phenomenon for conducting intracellular signals to various metastasis-related molecules (Tai et al., 2015). It was found that suppression of FAK in CCA cells by cucurbitacin B was accompanied with down-regulation of MMP-9 which subsequently resulted in decrease MMP-9 level. Additionally, ICAM-1 and VEGF were also down-regulated after cucurbitacin $\mathrm{B}$ exposure. Collectively, these findings indicated that anti-metastasis potential of cucurbitacin B against CCA cells was mediated, at least in part, through inhibition of FAK protein. Besides FAK, it has been reported that cucurbitacin B also exerted its anti-metastasis effect via modulating other signaling pathways including HippoYAP signaling pathway in colorectal cancer cells (Chai et al., 2018) and JAK2/STAT3 and MAPK pathways in osteosarcoma cells (Zhang et al., 2017). It is therefore likely that other intracellular signaling cascades might also be participating in the anti-metastasis activity of cucurbitacin B on CCA cells, which needs further investigation study.

In conclusion, cucurbitacin B possesses anti-metastasis property against CCA cells by blocking of FAK activation, suppressing metastatic phenotypes and down-regulating metastasis-associated molecules. These findings support the potential of cucurbitacin B to be developed as a novel therapeutic agent for CCA metastasis therapy.

\section{Acknowledgements}

This research was supported by the grant from Khon Kaen University, Thailand (6200011003), the Invitation Research grant from Faculty of Medicine, Khon Kaen University, Thailand (IN63252), and partially funded by a grant from the Office of the Higher Education Commission of Thailand. Putthaporn Kaewmeesri was supported by a Commission on Higher Education Ph.D. Scholarship. The authors would like to thank Mr. Gurdeep Singh, for editing the manuscript via the English Editing Publication Clinic, Faculty of Medicine, Khon Kaen University, Thailand.

\section{References}

Barni MV, Carlini MJ, Cafferata EG, et al (2012). Carnosic acid inhibits the proliferation and migration capacity of human colorectal cancer cells. Oncol Rep, 27, 1041-8.

Bielenberg DR, Zetter BR (2015). The contribution of angiogenesis to the process of metastasis. Cancer $J, \mathbf{2 1}$, 267-73.

Chai Y, Xiang K, Wu Y, et al (2018). Cucurbitacin B inhibits the
Hippo-YAP signaling pathway and exerts anticancer activity in colorectal cancer cells. Med Sci Monit, 24, 9251-8.

Charonpongsuntorn C, Piyasatit P, Muntham D, et al (2019). Clinical prognostic factors and treatment outcomes for the survival of patients with cholangiocarcinoma in the Eastern region of Thailand. Asian Pac J Cancer Care, 4, 101-5.

Curran S, Murray GI (2000). Matrix metalloproteinases: molecular aspects of their roles in tumour invasion and metastasis. Eur J Cancer, 36, 1621-30.

Doherty B, Nambudiri VE, Palmer WC (2017). Update on the diagnosis and treatment of cholangiocarcinoma. Curr Gastroenterol Rep, 19, 2.

Fouassier L, Marzioni M, Afonso MB, et al (2019). Signalling networks in cholangiocarcinoma: Molecularpathogenesis, targeted therapies and drug resistance. Liver Int, 39, 43-62.

Hauck CR, Sieg DJ, Hsia DA, et al (2001). Inhibition of focal adhesion kinase expression or activity disrupts epidermal growth factor-stimulated signaling promoting the migration of invasive human carcinoma cells. Cancer Res, 61, 7079-90.

Inan S, Hayran M (2019). Cell signaling pathways related to epithelial mesenchymal transition in cancer metastasis. Crit Rev Oncog, 24, 47-54.

Khansaard W, Techasen A, Namwat N, et al (2014). Increased EphB2 expression predicts cholangiocarcinoma metastasis. Tumour Biol, 35, 10031-41.

Klungsaeng S, Kukongviriyapan V, Prawan A, et al (2019). Cucurbitacin B induces mitochondrial-mediated apoptosis pathway in cholangiocarcinoma cells via suppressing focal adhesion kinase signaling. Naunyn Schmiedebergs Arch Pharmacol, 392, 271-8.

Klungsaeng S, Kukongviriyapan V, Prawan A, et al (2020). Targeted modulation of FAK/PI3K/PDK1/AKT and FAK/ p53 pathways by cucurbitacin $B$ for the antiproliferation effect against human cholangiocarcinoma cells. Am J Chin Med, 48, 1475-89.

Lee BY, Timpson P, Horvath LG, et al (2015). FAK signaling in human cancer as a target for therapeutics. Pharmacol Ther, 146, 132-49.

Liang J, Zhang XL, Yuan JW, et al (2019). Cucurbitacin B inhibits the migration and invasion of breast cancer cells by altering the biomechanical properties of cells. Phytother Res, 33, 618-30.

Luo WW, Zhao WW, Lu JJ, et al (2018). Cucurbitacin B suppresses metastasis mediated by reactive oxygen species (ROS) via focal adhesion kinase (FAK) in breast cancer MDA-MB-231 cells. Chin J Nat Med, 16, 9-10.

Mahdavi M, Shokrgozar M, Sardari S, et al (2017). Disruption of FGF2-Heparin-FGFR1 complex formation by antiangiogenic small molecule found in silico. Asian Pac J Cancer Biol, 2, 31-9.

Masraksa W, Tanasawet S, Hutamekalin P, et al (2020). Luteolin attenuates migration and invasion of lung cancer cells via suppressing focal adhesion kinase and non-receptor tyrosine kinase signaling pathway. Nutr Res Pract, 14, 127-33.

Paschos KA, Canovas D, Bird NC (2009). The role of cell adhesion molecules in the progression of colorectal cancer and the development of liver metastasis. Cell Signal, 21, 665-74.

Patlolla JM, Rao CV (2012). Triterpenoids for cancer prevention and treatment: current status and future prospects. Curr Pharm Biotechnol, 13, 147-55.

Peng X, Ueda H, Zhou H, et al (2004). Overexpression of focal adhesion kinase in vascular endothelial cells promotes angiogenesis in transgenic mice. Cardiovasc Res, 64, 421-30.

Pitchakarn P, Chewonarin T, Ogawa K, et al (2013). Ellagic acid inhibits migration and invasion by prostate cancer cell lines. Asian Pac J Cancer Prev, 14, 2859-63. 
Saensa-Ard S, Leuangwattanawanit S, Senggunprai L, et al (2017). Establishment of cholangiocarcinoma cell lines from patients in the endemic area of liver fluke infection in Thailand. Tumour Biol, 39, 1010428317725925.

Schlaepfer DD, Mitra SK, Ilic D (2004). Control of motile and invasive cell phenotypes by focal adhesion kinase. Biochim Biophys Acta, 1692, 77-102.

Sun C, Cui H, Yang H, et al (2015). Anti-metastatic effect of jolkinolide $\mathrm{B}$ and the mechanism of activity in breast cancer MDA-MB-231 cells. Oncol Lett, 10, 1117-22.

Taglia L, Matusiak D, Matkowskyj KA, et al (2007). Gastrin-releasing peptide mediates its morphogenic properties in human colon cancer by upregulating intracellular adhesion protein-1 (ICAM-1) via focal adhesion kinase. Am J Physiol Gastrointest Liver Physiol, 292, G182-90.

Tai YL, Chen LC, Shen TL (2015). Emerging roles of focal adhesion kinase in cancer. Biomed Res Int, 2015, 690690.

Tuponchai P, Kukongviriyapan V, Prawan A, et al (2019). Myricetin ameliorates cytokine-induced migration and invasion of cholangiocarcinoma cells via suppression of STAT3 pathway. J Cancer Res Ther, 15, 157-63.

Welch DR, Hurst DR (2019). Defining the hallmarks of metastasis. Cancer Res, 79, 3011-27.

Zakaria KN, Amid A, Zakaria Z, et al (2019). Anti-proliferative activity of triterpenes isolated from Clinicanthus nutans on Hep-G2 liver cancer cells. Asian Pac J Cancer Prev, 20, 563-7.

Zhang ZR, Gao MX, Yang K (2017). Cucurbitacin B inhibits cell proliferation and induces apoptosis in human osteosarcoma cells via modulation of the JAK2/STAT3 and MAPK pathways. Exp Ther Med, 14, 805-12.

Zheng S, Zhu Y, Zhao Z, et al (2017). Liver fluke infection and cholangiocarcinoma: a review. Parasitol Res, 116, 9-11.

\section{c) (7) (8)}

This work is licensed under a Creative Commons AttributionNon Commercial 4.0 International License. 